\title{
Suppression of Ischemic and Reperfusion Ventricular Arrhythmias by Inhalational Anesthetic-Induced Preconditioning in the Rat Heart
}

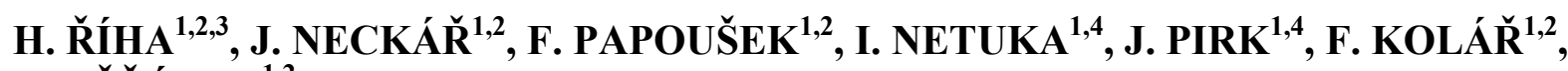 \\ B. OŠŤÁDAL ${ }^{1,2}$
}

${ }^{1}$ Centre for Cardiovascular Research, Prague, Czech Republic, ${ }^{2}$ Institute of Physiology, Academy of Sciences of the Czech Republic, Prague, Czech Republic, ${ }^{3}$ Department of Anesthesiology and Intensive Care Medicine, Institute for Clinical and Experimental Medicine, Prague, Czech Republic, ${ }^{4}$ Department of Cardiovascular Surgery, Institute for Clinical and Experimental Medicine, Prague, Czech Republic

Received December 13, 2010

Accepted May 3, 2011

\begin{abstract}
Summary
Inhalational anesthetic-induced preconditioning (APC) has been shown to reduce infarct size and attenuate contractile dysfunction caused by myocardial ischemia. Only a few studies have reported the effects of APC on arrhythmias during myocardial ischemia-reperfusion injury, focusing exclusively on reperfusion. Accordingly, the aim of the present study was to examine the influence of APC on ventricular arrhythmias evoked by regional no-flow ischemia. APC was induced in adult male Wistar rats by 12 -min exposures to two different concentrations ( 0.5 and $1.0 \mathrm{MAC}$ ) of isoflurane followed by 30 -min wash-out periods. Ventricular arrhythmias were assessed in the isolated perfused hearts during a 45-min regional ischemia and a subsequent 15-min reperfusion. Myocardial infarct size was determined after an additional $45 \mathrm{~min}$ of reperfusion. The incidence, severity and duration of ventricular arrhythmias during ischemia were markedly reduced by APC. The higher concentration of isoflurane had a larger effect on the incidence of ventricular fibrillation than the lower concentration. The incidence of ventricular tachycardia and reversible ventricular fibrillation during reperfusion was also significantly reduced by APC; the same was true for myocardial infarct size. In conclusion, we have shown that preconditioning with isoflurane confers profound protection against myocardial ischemia- and reperfusion-induced arrhythmias and lethal myocardial injury.
\end{abstract}

\section{Key words}

Anesthetic-induced preconditioning - Isoflurane - Ischemiareperfusion injury $\bullet$ Ventricular arrhythmias $\bullet$ Rat heart

\section{Corresponding author}

H. Říha, Department of Anesthesiology and Intensive Care Medicine, Institute for Clinical and Experimental Medicine, Vídeňská 1958/9, 14021 Prague, Czech Republic. Fax: +42026136 2799. E-mail: hynek.riha@ikem.cz

It has been repeatedly shown that inhalational anesthetics have cardioprotective properties (De Hert et al. 2009). Nearly all studies investigating preconditioning with inhalational anesthetics, i.e., brief exposure to inhalational anesthetics with a subsequent wash-out period before prolonged ischemic insult (anestheticinduced preconditioning, APC), have demonstrated improved myocardial resistance to a subsequent coronary artery occlusion, comparable with classical ischemic preconditioning (IPC) (Murry et al. 1986). The measured end-points of injury caused by myocardial ischemia in APC experiments have shown decreased myocardial infarct size (Kersten et al. 1997) and better post-ischemic recovery of contractile function (Kevin et al. 2003). Subsequent studies have revealed the similarity between APC and IPC in activated subcellular pathways (Stadnicka et al. 2007). 
Life-threatening arrhythmias are known to occur during myocardial ischemia and subsequent reperfusion. It has been observed that IPC offers significant protection against ischemia- (Kolar and Parratt 1997, Ravingerova et al. 2009) and reperfusion-induced arrhythmias (Hagar et al. 1991). One of the involved mechanisms seems to be an attenuation of conduction delay (Hong et al. 1999). However, only a few studies reported the influence of APC on arrhythmias during myocardial ischemiareperfusion, and these have focused exclusively on reperfusion (Kevin and Novalija 2008).

The main objective of our study was to investigate the effect of APC on the incidence, severity and duration of ventricular arrhythmias in the isolated rat heart induced by (i) myocardial regional no-flow ischemia and (ii) subsequent reperfusion. Myocardial infarct size was determined as the end-point of irreversible cell injury.

The experimental protocol was approved by the Animal Care and Use Committee of the Institute of Physiology, Academy of Sciences of the Czech Republic. Experiments were conducted in accordance with the Guide for the Care and Use of Laboratory Animals published by the US National Institutes of Health (NIH publication No. 85-23, revised 1996). Adult male Wistar rats (body weight [BW] 300-375 g) were randomly divided into three groups:

1) APC with 0.5 of minimal alveolar concentration (MAC) of isoflurane $(n=17)$,

2) APC with 1.0 MAC of isoflurane $(n=17)$ and

$3)$ control group without APC $(n=20)$.

APC was induced by a 12-min exposure of rats to a given inspiratory concentration of isoflurane in the air $\left(\mathrm{FiO}_{2}\right.$ 0.21) using a calibrated closed-box system with a variable bypass vaporizer. After exposure, the rats were returned to separate cages for a 30-min wash-out period.

Subsequently, after intraperitoneal administration of sodium pentobarbital $(60 \mathrm{mg} / \mathrm{kg})$, the hearts were excised and perfused according to Langerdorff under non-recirculating conditions using modified Krebs-Henseleit solution with reduced potassium and elevated calcium levels to promote arrhythmogenesis containing (in mmol/l): $\mathrm{NaCl} 118.0$; $\mathrm{KCl} 3.2 ; \mathrm{CaCl}_{2}$ 2.5; $\mathrm{MgSO}_{4}$ 1.2; $\mathrm{NaHCO}_{3}$ 25.0; $\mathrm{KH}_{2} \mathrm{PO}_{4}$ 1.2; glucose 7.0; Na-pyruvate 2.0. The perfusion solution was saturated with $95 \% \mathrm{O}_{2}$ and $5 \% \mathrm{CO}_{2}$ $\left(\mathrm{pH} 7.4 ; 37^{\circ} \mathrm{C}\right.$ ), and the coronary flow was adjusted to $10 \mathrm{ml} / \mathrm{min} / \mathrm{g}$ of heart weight (HW) calculated from the HW/BW ratio equation (Asemu et al. 1999). Mean perfusion pressure was measured in an aortic cannula. Epicardial electrogram was recorded with platinum electrodes attached to the right atrium and the apex of the heart.

After a 25-min stabilization period, the hearts were subjected to 45 -min regional ischemia, produced by the occlusion of the left anterior descending coronary artery, and subsequent 60-min reperfusion, which is sufficient to assess infarct size (Ferrera et al. 2009).

Recorded epicardial electrograms, heart rate (HR) values and perfusion pressure values were analyzed using custom-designed software. The severity of ventricular arrhythmias during ischemia was assessed according to the Lambeth Conventions (Walker et al. 1988) and evaluated by an arrhythmia score (from 1 to 5) depending on the incidence of the most severe form of arrhythmia that occurred in each individual heart. Ventricular arrhythmias occurring during the first $15 \mathrm{~min}$ of reperfusion were analyzed in a similar manner. The hearts exhibiting sustained ventricular fibrilation (VF; $>2$ min duration) were excluded from the analysis.

After the 60-min reperfusion period, the hearts were arrested in diastole. The area at risk (AR) and the size of infarct area (IS) were delineated by staining with $5 \%$ potassium permanganate and $1 \%$ triphenyltetrazolium chloride as described earlier (Neckar et al. 2003). The hearts were cut transversely into $1 \mathrm{~mm}$ thick slices, the right ventricular free wall was separated, and both sides of slices were photographed. The sizes of the IS, AR and left ventricle (LV) were determined by a computerized planimetric method.

Data are expressed as means \pm S.E.M. Differences in the number of premature ventricular complexes (PVCs) and the numbers of episodes of ventricular tachycardia (VT) and VF among the groups were compared by a Kruskal-Wallis test with subsequent Dunn's test. The incidences of VT and VF were examined by Fisher's exact test. The comparisons of differences in all other parametric variables were made by one-way analysis of variance (ANOVA) or repeated measures ANOVA. If the findings from the ANOVA were significant, Newman-Keuls analysis was used. Differences were considered statistically significant when $P<0.05$.

Baseline values of HR were comparable among the control and two APC groups (259 \pm 4 vs. $256 \pm 4$ and $256 \pm 4$ beats/min) and remained unaffected during experiments. Perfusion pressure significantly increased after coronary artery occlusion and did not change 
significantly during ischemia; after the beginning of reperfusion, it returned to pre-ischemic values.

The severity of ventricular arrhythmias induced by ischemia was significantly less pronounced in the hearts preconditioned by APC than in the control hearts. APC significantly reduced the total number of PVCs (Fig. 1A) as well as of PVCs occurring as salvos and VT (Table 1). VT was the predominant type of ischemiainduced arrhythmia in all of the experimental groups. Whereas the incidence of VT did not differ among the groups, preconditioned hearts exhibited a significantly lower number of PVCs occurring as VT with a lower number of VT episodes and shorter total VT duration. The incidence of reversible VF was reduced by APC, but this effect was significant only at the higher concentration of isoflurane; the same was true for the incidence of sustained VF. However, the number of episodes and total duration of reversible VF were significantly decreased in both APC groups compared to the control group. The overall suppression of ischemic ventricular arrhythmias by APC expressed as the significant reduction in arrhythmia score values is shown in Fig. 1B. Ischemia induced ventricular ectopic activity with the maximum occurring between 10 and $20 \mathrm{~min}$. APC affected the temporal profile (distribution of PVCs over 45-min ischemia) of ventricular arrhythmias (Fig. 1C); the occurrence of the first PVC after the beginning of ischemia was markedly delayed in both APC groups compared to the control group $(8.4 \pm 0.2$ and $8.5 \pm 0.2 \mathrm{~min}$ vs. $3.0 \pm 0.3 \mathrm{~min} ; P<0.0001)$, and the median of PVC distribution in the preconditioned hearts occurred significantly later $(15.2 \pm 0.1$ and $15.2 \pm 0.1$ vs. $14.5 \pm 0.1$ $\min ; P=0.002$ ).

During the first $15 \mathrm{~min}$ of reperfusion, the incidence of VT (50.0 and 52.9 vs. $91.7 \% ; P<0.05)$ and reversible VF (18.8 and 17.6 vs. $58.3 \% ; P<0.05)$ was markedly attenuated by APC. The reduction in the incidence of sustained VF did not reach statistical significance in any APC group. The overall suppression of reperfusion-induced arrhythmias expressed as arrhythmia score showed significant influence of APC (Fig. 1D).
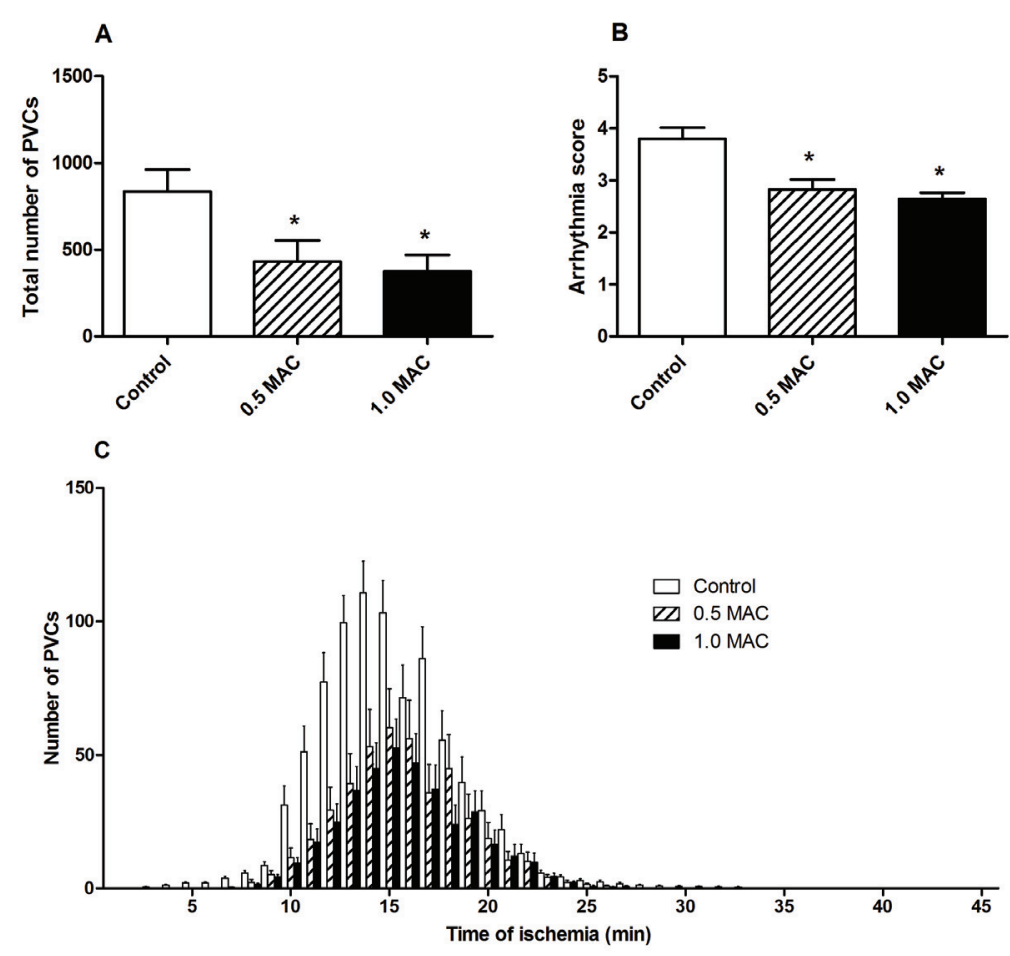

$$
\text { D }
$$
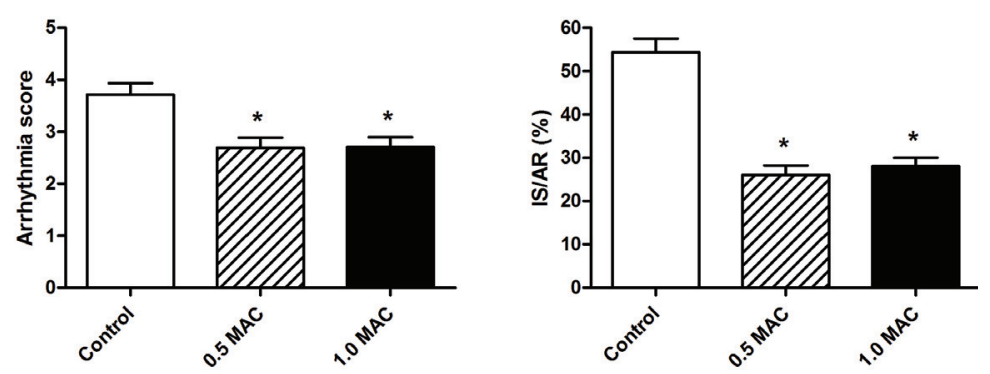

Fig. 1. The influence of anesthetic-induced preconditioning with 0.5 and 1.0 MAC of isoflurane on ventricular arrhythmias and infarct size in rat hearts. A: Total number of premature ventricular complexes (PVCs) during 45-min regional ischemia. B: Arrhythmia score over 45-min regional ischemia. C: Distribution of PVCs during 45-min regional ischemia. D: Arrhythmia score over the first $15 \mathrm{~min}$ of reperfusion. E: The infarct size (IS) normalized to the area at risk (AR). Values are means \pm S.E.M. ${ }^{*} P<0.05$ vs. the control group. 
Table 1. Premature ventricular complexes occurring as singles, salvos and ventricular tachycardia, incidence of ventricular tachycardia and ventricular fibrillation, number of episodes and total duration of ventricular tachycardia and reversible ventricular fibrillation during $45 \mathrm{~min}$ of regional ischemia in control and isoflurane-preconditioned rat hearts.

\begin{tabular}{|c|c|c|c|c|c|c|c|}
\hline \multirow[t]{2}{*}{ Group } & \multirow[t]{2}{*}{$\mathbf{n}$} & \multicolumn{3}{|c|}{ Number of PVCs } & \multicolumn{3}{|c|}{ Incidence (\%) } \\
\hline & & Singles & Salvos & VT & VT & VFr & VFs \\
\hline Control & 20 & $143 \pm 26$ & $131 \pm 18$ & $563 \pm 120$ & 92.9 & 35.7 & 30.0 \\
\hline$A P C 0.5 M A C$ & 17 & $130 \pm 23$ & $70 \pm 22^{*}$ & $232 \pm 106^{*}$ & 62.5 & 6.3 & 5.9 \\
\hline$A P C 1.0 M A C$ & 17 & $113 \pm 24$ & $62 \pm 16^{*}$ & $201 \pm 92^{*}$ & 64.7 & $0^{*}$ & $0^{*}$ \\
\hline \multirow[t]{2}{*}{ Group } & \multirow[t]{2}{*}{$\mathbf{n}$} & \multicolumn{3}{|c|}{ Number of episodes } & \multicolumn{2}{|c|}{ Duration (s) } & n VFs \\
\hline & & $\mathbf{V}$ & & VFr & VT & VFr & \\
\hline Control & 20 & $26.6=$ & & $1.7 \pm 0.8$ & $51.8 \pm 11.5$ & $16.5 \pm 6.8$ & 6 \\
\hline$A P C 0.5 M A C$ & 17 & $11.0=$ & & $0.06 \pm 0.06^{*}$ & $21.0 \pm 9.8^{*}$ & $0.8 \pm 0.8^{*}$ & 1 \\
\hline$A P C 1.0 M A C$ & 17 & $9.0 \pm$ & & $0^{*}$ & $18.1 \pm 8.2^{*}$ & $0^{*}$ & 0 \\
\hline
\end{tabular}

Values are means \pm S.E.M. ${ }^{*} P<0.05$ vs. the control group. APC, anesthetic-induced preconditioning with isoflurane; MAC, minimal alveolar concentration; $n$, number of animals; PVCs, premature ventricular complexes; VT, ventricular tachycardia; VFr, reversible ventricular fibrillation; VFs, sustained ventricular fibrillation; $n$ VFs, number of hearts exhibiting sustained ventricular fibrillation (excluded from the analysis with the exception of the incidence of VFs).

The area at risk normalized to the size of the left ventricle $(\mathrm{AR} / \mathrm{LV})$ did not differ among the control and two APC groups ( $42.2 \pm 2.3$ vs. $43.1 \pm 2.1$ and $40.9 \pm 2.5 \%)$. The infarct size normalized to the AR (IS/AR) was significantly decreased in both APC groups (Fig. 1E).

The main finding of the present study is the significant suppression of ventricular arrhythmias during myocardial regional no-flow ischemia after APC induced by isoflurane in an in vivo/in vitro rat model. To the best of our knowledge, the present study is the first to investigate the influence of APC on ventricular arrhythmias during myocardial regional ischemia. Previous experimental studies pointed to the possibility of an antiarrhythmic effect of APC and demonstrated reductions in the incidence of VF during reperfusion after global ischemia (Novalija et al. 1999, Kevin et al. 2003). These studies primarily examined other end-points of cardiac injury, and the antiarrhythmic effect was observed as a side result. The first study primarily investigating the antiarrhythmic protection by APC documented a prolonged time to the onset of arrhythmias and a decrease in VF incidence during 120-min reperfusion after 30-min global and regional ischemia when APC was induced by the administration of 0.5 and 1.0 MAC of isoflurane for $30 \mathrm{~min}$ (Kevin and Novalija 2008). The authors, however, did not study the ischemic phase preceding the reperfusion.

There were no differences in the cardioprotection between 0.5 and 1.0 MAC of isofluraneinduced APC with one exception: the higher isoflurane concentration reduced the incidence of ischemia-induced VF (both reversible and sustained) more than the lower concentration. However, the temporal profile of ischemic arrhythmias did not differ between APC groups. Other dose-related differences in isoflurane-induced preconditioning were observed in various experiments (Kehl et al. 2002, Obal et al. 2005).

A possible limitation of our study is that we used an in vivo/in vitro model with APC induced by isoflurane exposure in vivo and subsequent in vitro assessment of ventricular arrhythmias in the isolated hearts. The main reason for using this model has been the conflicting results of the studies reporting cardioprotective effects of in vitro pre-ischemic administration of isoflurane (Martini et al. 2001). Furthermore, an in vivo/in vitro model was used by other groups exploring APC (Lutz and Liu 2006). Administration of inhalational anesthetics in vivo causes alterations in the cardiovascular and respiratory systems (Preckel and Bolten 2005) and thus carries the risk of possible influence on the subsequent in vitro experiment. However, it has been documented that concentrations $\leq 1$ MAC of isoflurane did not significantly influence hemodynamics and blood gases (Eger 1981). Previous studies have also found that even a concentration as low as $0.25 \mathrm{MAC}$ of isoflurane is sufficient to produce APC (Kehl et al. 2002). Thus, low 
concentrations of isoflurane for a short time interval were used in our experiment to induce APC, avoiding at the same time significant cardiovascular and respiratory changes.

In conclusion, we demonstrated significant antiarrhythmic effect of APC induced by 0.5 and 1.0 MAC of isoflurane on ventricular arrhythmias produced by regional myocardial ischemia and subsequent reperfusion in the rat hearts. APC changed the temporal profile of ischemic arrhythmias with significant delay in the occurrence of the first and median PVC. Furthermore, we confirmed, in accordance with previous experimental studies, a significant reduction in myocardial infarct size after ischemia-reperfusion by APC (Kersten et al. 1997, Kehl et al. 2002, Kevin et al. 2003). These facts suggest that inhalational anesthetic-induced preconditioning has not only the frequently reported protective effect against lethal myocardial injury but also a significant antiarrhythmic effect.

\section{Conflict of Interest}

There is no conflict of interest.

\section{Acknowledgements}

The study was supported by the grants from the Ministry of Education of the Czech Republic (MSMT 1M0510) and the Grant Agency of the Academy of Sciences of the Czech Republic (IAA 501110901). The authors acknowledge the support of Ondrej Szarszoi, MD, $\mathrm{PhD}$ and Jiri Maly $\mathrm{MD}, \mathrm{PhD}$ with conduct of study, and the support of Ales Brezina, MD, CSc and Ladislav Hess, $\mathrm{MD}$, DrSc with study design.

\section{References}

ASEMU G, PAPOUSEK F, OSTADAL B, KOLAR F: Adaptation to high altitude hypoxia protects the rat heart against ischemia-induced arrhythmias. Involvement of mitochondrial K(ATP) channel. J Mol Cell Cardiol 31: 1821$1831,1999$.

DE HERT SG, PRECKEL B, SCHLACK WS: Update on inhalational anaesthetics. Curr Opin Anaesthesiol 22: 491$495,2009$.

EGER EI 2ND: Isoflurane: a review. Anesthesiology 55: 559-576, 1981.

FERRERA R, BENHABBOUCHE S, BOPASSA JC, LI B, OVIZE M: One hour reperfusion is enough to assess function and infarct size with TTC staining in Langendorff rat model. Cardiovasc Drugs Ther 23: 327-331, 2009.

HAGAR JM, HALE SL, KLONER RA: Effect of preconditioning ischemia on reperfusion arrhythmias after coronary artery occlusion and reperfusion in the rat. Circ Res 68: 61-68, 1991.

HONG K, KUSANO KF, MORITA H, FUJIMOTO Y, WANG X, YAMANARI H, OHE T: Attenuation of conduction delay by ischemic preconditioning reduces ischemia-induced ventricular arrhythmias. Acta Med Okayama 53: 233-238, 1999.

KEHL F, KROLIKOWSKI JG, MRAOVIC B, PAGEL PS, WARLTIER DC, KERSTEN JR: Is isoflurane-induced preconditioning dose related? Anesthesiology 96: 675-680, 2002.

KERSTEN JR, SCHMELING TJ, PAGEL PS, GROSS GJ, WARLTIER DC: Isoflurane mimics ischemic preconditioning via activation of K(ATP) channels: reduction of myocardial infarct size with an acute memory phase. Anesthesiology 87: 361-370, 1997.

KEVIN LG, KATZ P, CAMARA AK, NOVALIJA E, RIESS ML, STOWE DF: Anesthetic preconditioning: effects on latency to ischemic injury in isolated hearts. Anesthesiology 99: 385-391, 2003.

KEVIN LG, NOVALIJA E: Anesthetic preconditioning decreases arrhythmias and improves regional conduction in isolated hearts. J Cardiothorac Vasc Anesth 22: 217-224, 2008.

KOLAR F, PARRATT JR: Antiarrhythmic effect of ischemic preconditioning in hearts of spontaneously hypertensive rats. Exp Clin Cardiol 2: 124-127, 1997.

LUTZ M, LIU H: Inhaled sevoflurane produces better delayed myocardial protection at 48 versus 24 hours after exposure. Anesth Analg 102: 984-990, 2006.

MARTINI N, PRECKEL B, THAMER V, SCHLACK W: Can isoflurane mimic ischaemic preconditioning in isolated rat heart? Br J Anaesth 86: 269-271, 2001. 
MURRY CE, JENNINGS RB, REIMER KA: Preconditioning with ischemia: a delay of lethal cell injury in ischemic myocardium. Circulation 74: 1124-1136, 1986.

NECKAR J, SZARSZOI O, HERGET J, OSTADAL B, KOLAR F: Cardioprotective effect of chronic hypoxia is blunted by concomitant hypercapnia. Physiol Res 52: 171-175, 2003.

NOVALIJA E, FUJITA S, KAMPINE JP, STOWE DF: Sevoflurane mimics ischemic preconditioning effects on coronary flow and nitric oxide release in isolated hearts. Anesthesiology 91: 701-712, 1999.

OBAL D, WEBER NC, ZACHAROWSKI K, TOMA O, DETTWILER S, WOLTER JI, KRATZ M, MULLENHEIM J, PRECKEL B, SCHLACK W: Role of protein kinase C-epsilon (PKCepsilon) in isoflurane-induced cardioprotection. Br J Anaesth 94: 166-173, 2005.

PRECKEL B, BOLTEN J: Pharmacology of modern volatile anaesthetics. Best Pract Res Clin Anaesthesiol 19: 331$348,2005$.

RAVINGEROVA T, MATEJIKOVA J, PANCZA D, KOLAR F: Reduced susceptibility to ischemia-induced arrhythmias in the preconditioned rat heart is independent of PI3-kinase/Akt. Physiol Res 58: 443-447, 2009.

STADNICKA A, MARINOVIC J, LJUBKOVIC M, BIENENGRAEBER MW, BOSNJAK ZJ: Volatile anestheticinduced cardiac preconditioning. J Anesth 21: 212-219, 2007.

WALKER MJ, CURTIS MJ, HEARSE DJ, CAMPBELL RW, JANSE MJ, YELLON DM, COBBE SM, COKER SJ, HARNESS JB, HARRON DW, HIGGINS AJ, JULIAN DG, LAB MJ, MANNING AS, NORTHOVER BJ, PARRATT JR, RIEMERSMA RA, RIVA E, RUSSELL DC, SHERIDAN DJ, WINSLOW E, WOODWARD B: The Lambeth Conventions: guidelines for the study of arrhythmias in ischaemia, infarction, and reperfusion. Cardiovasc Res 22: 447-455, 1988. 